\title{
Practical Algorithm for large diameter pile tip bearing capacity based on displacement control
}

\author{
RUAN Xiang ${ }^{1, a}$ \\ ${ }^{1}$ Shenzhen Institute of Building Research Co., Ltd. 518049, China \\ arx_gongkan@126.com
}

\begin{abstract}
Key words:Large diameter pile; tip bearing capacity; allowable displacement; Mindlin solution Abstract:Based on the Mindlin solution of stress distribution under internal point load in semi infinite space, this paper presents a new Practical Algorithm for the tip bearing capacity of large diameter pile based on displacement control. First, use the Mindlin solution under circular uniform load to derive the additional stress formula of the large diameter pile bottom. Then, define the final settlement formula for the large diameter pile base on the single pile settlement formula form the code. Finally, inverse compute additional stress of pile bottom according to the pile settlement allowable value, and then conform the tip bearing capacity of large diameter pile. Compared to the engineering examples, the calculation results of this paper match well.
\end{abstract}

\section{Introduction}

According to the code [1], the large diameter pile diameter is greater or equal to $800 \mathrm{~mm}$. The application of large diameter pile is more and more extensive with the development of high-rise buildings. In order to improve the capacity of the large diameter pile, the pile bottom is often made in the form of bit expansion. Conform the tip capacity requires a static load test theoretically. But conditions are limited in practical engineering, the test method is difficult to achieve. Experience is often used to ascertain the tip capacity, and simplified induction to the reduction factor at last.

Many scholars launched a series of studies to conform the tip capacity of large diameter pile currently [2-5], the research results are reduction factors, and difference is the value. But factors are many that infect the tip capacity, such as belled diameter, embed depth, thickness of the bearing stratum, and whether there exist soft stratum and so on. To get the precise tip capacity, many factors above should be considered.

For normal consolidation foundation soil, settlement is mainly caused by additional stress generated by the additional load, the Mindlin solution is common formula for calculate additional stress generated by applied force in semi-infinite space. All those factors is comprehensive considerate, the paper derive the bottom additional stress formula with Mindlin solution. On this basis, we derive the large diameter pile final settlement formula according to the unidirectional layered compression summation method. Then, we could calculate the tip capacity based on the additional stress which is back calculation from the allowable value of settlement for large diameter pile.

\section{Basic solutions and its promotion for Mindlin solution}

\section{Basic solutions for Mindlin solution}

In study the performance of single pile, confirm the bearing capacity of large diameter pile, chosen some calculation methods for pile and sheet pile wall, get on load test of deep soil in pile's bottom and settlement analysis for pier, raft foundation and box foundation those embed depth is getting bigger and bigger, underground space develops and utilize, Mindlin solution(R.D. Mindlin, 1936) as a precondition to concentrated force work on the elastic half space inside is more reasonable in theoretical and computational results logically compared to Boussinesq solution(Boussinesq,1885) as a precondition to concentrated force work on the elastic half space face [6].

Mindlin [7](1936) take into account the direction of the load, there are two cases that 
perpendicular to the semi-infinite space surface and parallel to the semi-infinite space surface. In case to calculate the stress distribution of deep foundation under vertical load, only take into account the situation that the load work on perpendicular to the semi-infinite space surface. Assume there is a concentrated force $\mathrm{P}$ in the elastic half infinite space depth $\mathrm{h}$, as shown in Fig. $1 . \sigma_{\mathrm{z}}$ the vertical stress at any point from the surface depth $\mathrm{Z}$ (free surface of semi - infinite space) as shown in Eq.1:

$$
\begin{aligned}
\sigma_{z}= & \frac{P}{8 \pi(1-\mu)}\left[-\frac{(1-2 \mu)(z-h)}{R_{1}^{3}}+\frac{(1-2 \mu)(z-h)}{R_{2}^{3}}-\frac{3(z-h)^{3}}{R_{1}^{5}}\right. \\
& -\frac{3(3-4 \mu) z(z+h)^{2}-3 h(z+h)(5 z-h)}{R_{2}^{5}}-\frac{30 h z(z+h)^{3}}{R_{2}^{7}}
\end{aligned}
$$

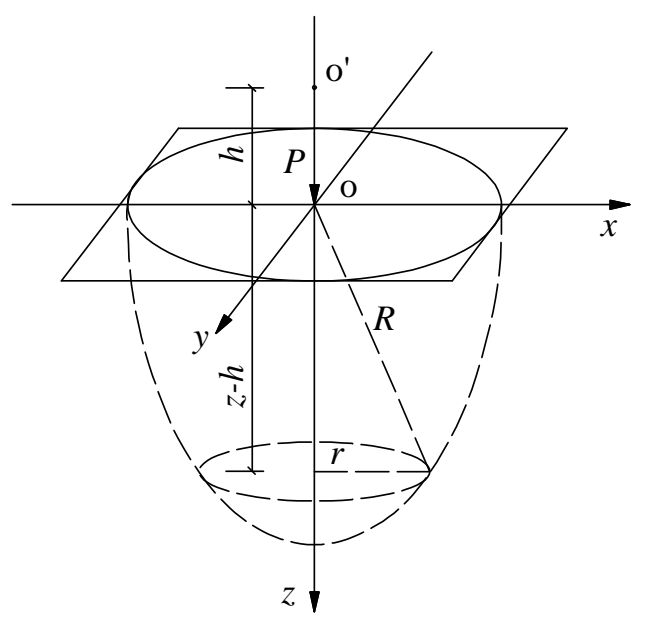

Fig.1 Hypothesis for Mindlin Solution

Promotion for Mindlin solution

No matter for common pile or belled pile foundation, acting inside the foundation is a circular contact surface, therefore the Mindlin solution can't be used to solve its stress distribution directly. The basic solutions must be extended to the situation of uniformly distributed load.

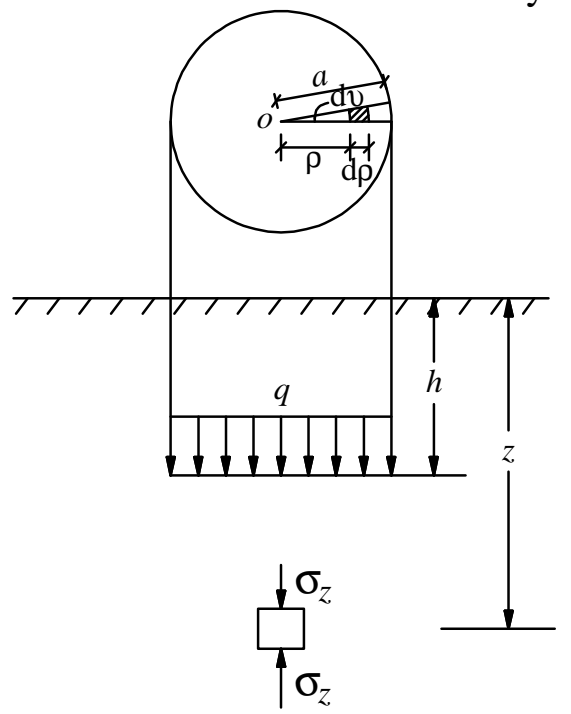

Fig.2 Circular Uniform Distributed Load in Soil

$\mathrm{Xu}$ Huai-ying[8] (1957) educed the Mindlin solution. Assume there is a circular uniformly distributed load work below the ground depth $\mathrm{h}$, the radius of circle is a, uniformly distributed load value is $\mathrm{q}$, as shown in Fig.2. In polar coordinate system, take the differential $\rho \mathrm{d} \rho \mathrm{d} \theta$ in circular uniformly distributed load, the load value in the micro area is $q \rho \mathrm{d} \rho \mathrm{d} \theta$. Vertical stress caused by 
$q \rho \mathrm{d} \rho \mathrm{d} \theta$ in the distance $\mathrm{Z}$ from $\mathrm{O}$ point to the ground could be presented as Eq.2:

$$
\begin{aligned}
\sigma_{z}=\frac{q}{4(1-\mu)}\left\{-2(1-\mu)+\frac{(1-2 \mu)(z-h)}{\sqrt{a^{2}+(z-h)^{2}}}+\frac{(1-2 \mu)(z-h)}{z+h}\right. \\
-\frac{(1-2 \mu)(z-h)}{\sqrt{a^{2}+(z+h)^{2}}}+\frac{(z-h)^{3}}{\left[a^{2}+(z-h)^{2}\right]^{3 / 2}}-\frac{(3-4 \mu) z}{z+h}+\frac{(3-4 \mu) z(z+h)^{2}}{\left[a^{2}+(z+h)^{2}\right]^{3 / 2}} \\
+\frac{h(5 z-h)}{(z+h)^{2}}-\frac{h(5 z-h)(z+h)}{\left[a^{2}+(z+h)^{2}\right]^{3 / 2}}-\frac{6 h z}{(z+h)^{2}}+\frac{6 h z(z+h)^{3}}{\left[a^{2}+(z+h)^{2}\right]^{5 / 2}}
\end{aligned}
$$

\section{Study on tip bearing capacity of large diameter pile}

Additional stress on the bottom of the large diameter pile

Mindlin solution and its promotion are based on the hypothesis of homogeneous elastic foundation. It will cause a greater deviation while use Mindlin solution to solve internal stress of foundation soil in limit State. The bearing capacity of large diameter pile is dominated by tip bearing capacity; the settlement value corresponding to ultimate bearing capacity may have been far more than the value for normal use. So the study of this article is the tip capacity corresponding to the allowed deformation by code [1].

In order to promote the capacity, large diameter pile is often made to enlarge tip. Because the belled tip existence, the impact on tip soil by side friction resistance can be ignored [9]. So the bearing capacity of large diameter pile can be divided into two parts, as shown in Fig.3. Tip capacity P2 is mainly in capacity of the large diameter pile. Make the belled tip Simplified as rigid board in semi-infinite space, the additional stress under the pile's bottom can be solved by Mindlin solution under the action of uniformly distributed load.

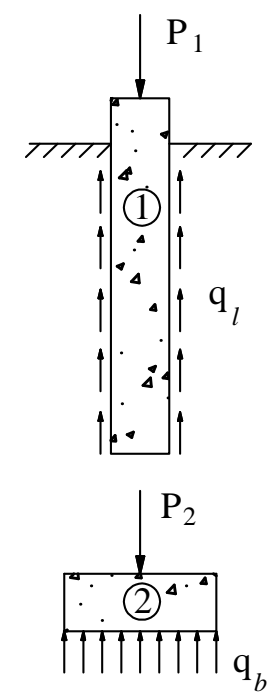

Fig.3 Force Exerting Mode for large diameter pile [9]

The final settlement of the large diameter pile

For normal consolidation foundation soil, settlement is mainly caused by additional stress generated by the additional load.

The description of the calculation formula for single pile settlement in code [1] is: The pile that the soil under the pile caps does not share the load, the additional stress caused by the pile below the tip plane could be calculated by Mindlin solution consider the effect of pile diameter in appendix F. Superimpose additional stress in stress calculation point caused by each pile in horizontal range of influence of settlement calculation point, calculate the settlement of the soil layer by unidirectional compression stratified sum, pile compression Se is included. The final settlement $\mathrm{S}$ of the pile is shown in Eq.3: 


$$
s=\psi \sum_{i=1}^{n} \frac{\sigma_{z i}}{E_{s i}} \Delta Z_{i}+s_{e}
$$

Specific meanings in formula see the literature [1]. For research convenience, settlement experience coefficient in formula takes 1.0.

For normal large diameter pile, the tip capacity is mainly, and the length is not very long, pile compression is ignored while calculate the settlement. Now, the Eq.3 could be simplified as:

$$
s=\sum_{i=1}^{n} \frac{\sigma_{z i}}{E_{s i}} \Delta Z_{i}
$$

\section{Engineering examples}

Inverse the tip capacity in normal

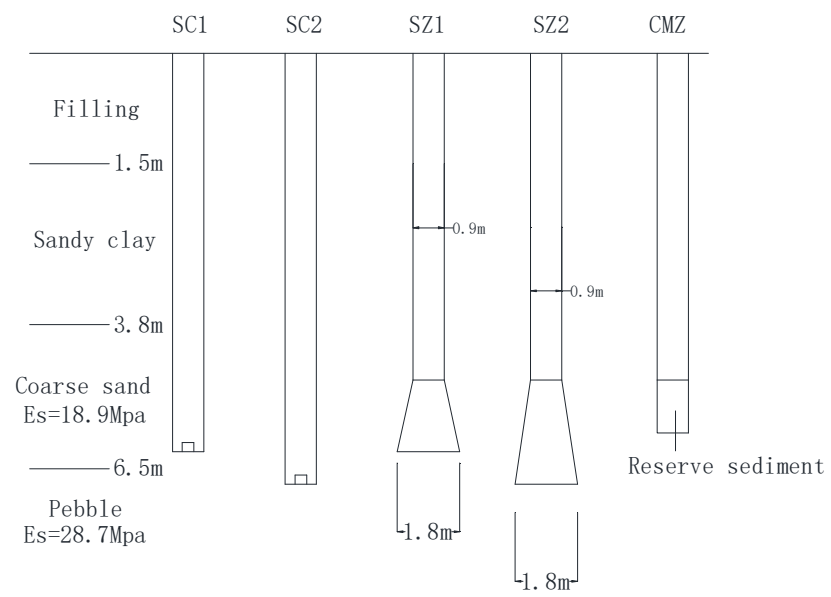

Fig.4 Field Test General Situation

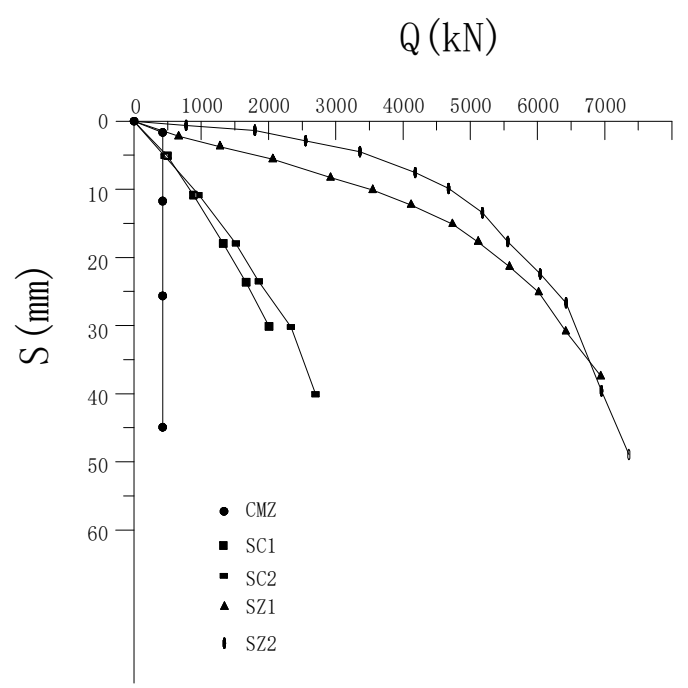

Fig.5 Q-s Curve

The large diameter pile is used in a project, the tip is belled. There are two sets of prototype piles, two sets of deep loading test and one set of pure friction pile in the test. The test profile is shown in Fig.4, the test results are shown in Fig.5. The result of pure friction pile test shows that limit value of side friction resistance is $400 \mathrm{kN}$ while Pile top settlement is $1.834 \mathrm{~mm}$. Take settlement $\mathrm{s} / \mathrm{D}=0.01$ for control objectives. When the settlement is $18 \mathrm{~mm}$, the capacity of SZ1 is $5450 \mathrm{kN}$, SZ2 is $5880 \mathrm{kN}$.Using the total bearing capacity of the pile minus the friction, and we could get the tip capacity. The results are shown in table 1:

Tab1. Calculation results in normal situation

\begin{tabular}{|c|c|c|c|c|c|}
\hline Numbering & $\sigma_{\mathrm{z}}[\mathrm{kPa}]$ & $q_{\mathrm{p}}[\mathrm{kPa}]$ & $\begin{array}{c}\text { Calculate tip bearing } \\
\text { capacity[kN] }\end{array}$ & $\begin{array}{c}\text { Measured tip bearing } \\
\text { capacity [kN] }\end{array}$ & $\begin{array}{c}\text { Deviation } \\
{[100 \%]}\end{array}$ \\
\hline SZ1 & 146 & 1878.65 & 4778.16 & 5050 & 5.38 \\
\hline SZ2 & 158 & 1986.54 & 5052.57 & 5480 & 7.80 \\
\hline
\end{tabular}

Inverse the tip capacity with soft substratum

The large diameter pile is used in a project, as shown in Fig.6: pile length in soil is L, the pile diameter is $0.8 \mathrm{~m}$, belled tip diameter $\mathrm{D}$ is $1.80 \mathrm{~m}$, the tip bearing stratum is claypan, and there is a soft substratum below the bearing substratum. There is $2.5 \mathrm{~m}$ from pile tip to substratum; the substratum thickness is $1.3 \mathrm{~m}$; the compression modulus ratio Es1/ E s2 of bearing substratum and substratum is 3 . The settlement is $\mathrm{s}$ while the tip capacity achieves the characteristic value of bearing capacity.

Suppose that the length of the pile in soil is $10.0 \mathrm{~m}$, the Poisson's ratio of bearing substratum and 
substratum is also 0.3 .

By Eq.4:

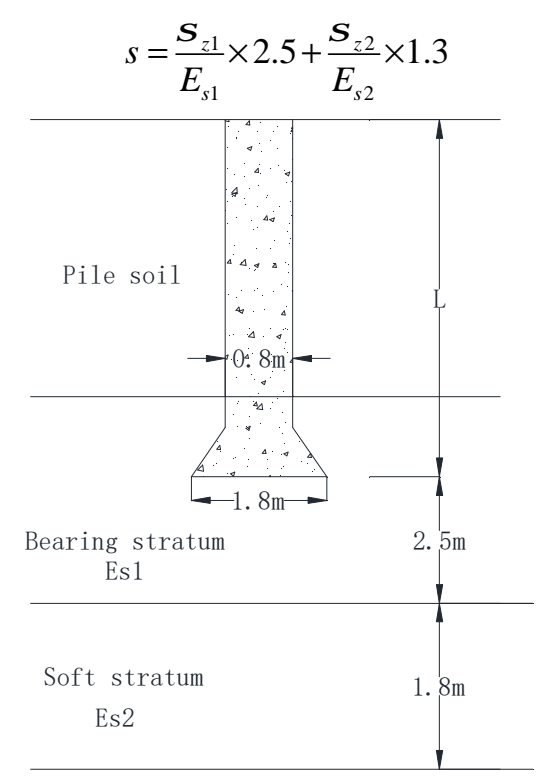

Fig.6 Large Diameter Pile with soft Substratum

By formula (2):

$$
\left.\begin{array}{l}
\sigma_{z 1}=0.503 q \\
\sigma_{z 2}=0.070 q
\end{array}\right\}
$$

For Es1/ Es2=3, combined formula (5), (6):

$$
s=\frac{0.503 q}{3 E_{s 2}} \times 2.5+\frac{0.070 q}{E_{s 2}} \times 1.3
$$

Assume there is no soft substratum below the tip, uniform load below the pile tip is $q^{\prime}$, by Eq.4:

Using Eq.2:

$$
\left.\begin{array}{c}
s=\frac{\sigma_{z 1}}{E_{s 1}} \times 2.5+\frac{\sigma_{z 2}}{E_{s 1}} \times 1.3 \\
\sigma_{z 1}=0.503 q^{\prime} \\
\sigma_{z 2}=0.070 q^{\prime}
\end{array}\right\}
$$

Combined Eq.8 and Eq. 9:

$$
s=\frac{0.503 q^{\prime}}{3 E_{s 2}} \times 2.5+\frac{0.070 q^{\prime}}{3 E_{s 2}} \times 1.3
$$

Simultaneous Eq.7 and Eq.10:

That is:

$$
\begin{gathered}
\frac{0.503 q^{\prime}}{3 E_{s 2}} \times 2.5+\frac{0.070 q^{\prime}}{3 E_{s 2}} \times 1.3=\frac{0.503 q}{3 E_{s 2}} \times 2.5+\frac{0.070 q}{E_{s 2}} \times 1.3 \\
q / q^{\prime}=0.88
\end{gathered}
$$

From Eq.12 we can see: the tip capacity is reduced by $12 \%$ due to the soft substratum in this project compared to the normal situation.

\section{Conclusions}

We solve the additional stress of the pile bottom for large diameter pile by using Mindlin solution. This paper presents a new Practical Algorithm for large diameter pile's tip bearing capacity based on displacement control according to the calculation formula of pile settlement. This method takes influences of belled diameter, embedded depth, thickness of bearing, soft substratum, physical and mechanical properties etc. into account. The Feasibility and reasonableness of the algorithm in this paper is supported by the engineering examples. 


\section{References}

[1] Industry Standard of the People's Republic of China. Technical code for building pile foundations (JGJ 94-2008). Beijing: China Architecture \& Building Press (2003), p.54

[2] Yang Tong-xin.Calculation of end bearing capacity of large diameter bottom enlarged pile in finite thick soil layer [J].Metal Mine Design \& Construction, 1997, 29(2), p22-p25

[3] Liu Xiao-wen, Liu Zu-de. Analysis of End Bearing Capacity of Large Diameter Bottom-Enlarged Pile in Soft Substratum. Site Investigation Science and Technology, 2003, (1), p21-p23

[4] Zai Jin-zhang, etc. Behavior Analyzes of Belled Pile in Layer soil and its Construction Applications. Fujian Architecture \& Construction, 1994, p19-p24

[5]Guo Xing-yu. Numerical simulation for vertical bearing behavior of belled pier. Shanghai: Tongji University, 2007, p1-p12

[6] Wang Shi-jie, Zhang mei, Zhang Ji-zhan. On Mindlin stress formulas. Engineering mechanics, 2001, 18(6),p141-p147

[7] Mindlin, R.D., Force at a point in the interior of a semi-infinite solid. Physics, 1936, MAY, Vol.7, p195-p202

[8] Xu Huai-ying. Calculation of Vertical Pressures in Soil Beneath Loaded Areas Based on Mindlin Formula. Chinese Journal of Civil Engineering, 1957, (4)4, p485-p497

[9] Gu Bao-he. Determination of axial bearing capacity of belled-end pier by settlement observation. Beijing: China Architecture and Building Press, 1987, p35-p42 